Meta

Journal des tradlucteurs

Translators' Journal

\title{
Le traducteur arabe est-il prêt à relever le défi?
}

\section{Henri Van Hoof et Samia Barrada}

Volume 35, numéro 4, décembre 1990

URI : https://id.erudit.org/iderudit/001977ar

DOI : https://doi.org/10.7202/001977ar

Aller au sommaire du numéro

Éditeur(s)

Les Presses de l'Université de Montréal

ISSN

0026-0452 (imprimé)

1492-1421 (numérique)

Découvrir la revue

Citer cette note

Van Hoof, H. \& Barrada, S. (1990). Le traducteur arabe est-il prêt à relever le défi? Meta, 35(4), 795-795. https://doi.org/10.7202/001977ar d'utilisation que vous pouvez consulter en ligne.

https://apropos.erudit.org/fr/usagers/politique-dutilisation/ 


\section{BLOC-NOTES}

\section{LE TRADUCTEUR ARABE EST-IL PRÊT À RELEVER LE DÉFI ?}

Sous l'égide de la Fédération internationale des traducteurs (FIT) et de 1'UNESCO, une table ronde sur «les problèmes de la traduction professionnelle dans le monde arabe et l'apport de la technologie» s'est tenue les 1, 2, 3 juin 1989 à l'École supérieure Roi Fahd de traduction à Tanger (Maroc). Une rencontre réussie tant par le nombre que par la qualité des participants, tous des professionnels et spécialistes de la traduction, des terminologues et des chercheurs en traduction automatique venus des différents pays d'Europe et du monde arabe.

Et une manifestation unique aussi dans son genre, car, comme l'a souligné sa présidente, Anna Lilova, lors de la séance d'ouverture, c'est la première fois que la FIT organise une telle rencontre dans un pays arabe.

Les communications ont porté sur trois thèmes essentiels, chacune des journées étant consacrée à l'un d'eux : arabes.

- La situation du traducteur dans les pays

- Le traducteur arabe et la TAO (traduction assistée par ordinateur).

Les efforts des pays arabes en matière de terminologie.

Dans les pays arabes, la traduction constitue une tradition qui remonte aux Abbassides. Mais aujourd'hui encore elle ne fait toujours pas l'objet d'une réglementation précise.

Le rapport de $M$. Amid (UNESCO) a mis l'accent sur la nécessité de mettre en cuvre, avec le concours des institutions de traduction, une législation qui protégerait les droits du traducteur.

Appel auquel a répondu M. René Haeseryn (secrétaire général de la FIT) qui, dans son exposé «La FIT et son rôle primordial dans le mouvement de la traduction dans le monde» a rappelé les objectifs humanistes de la Fédération et son rôle dans la protection des droits du traducteur.

Protéger le traducteur mais aussi le préparer aux grandes options de l'avenir, telle a été la préoccu pation majeure qui s'est dégagée de ces réunions de travail.

Traduire est un métier qui suppose un apprentissage selon une méthodologie réfléchie, ont dit en substance les divers intervenants. Il convient donc de mettre au point une formation appropriée aux besoins et de l'harmoniser dans les différents pays arabes.

De plus, aujourd'hui, grâce aux nouvelles technologies, le métier de traducteur voit son profil se modifier. Les choses évoluent vite.

Une présentation succincte par Véronica Lawson (FIT) des différents systèmes (machines à traduire avec ou sans assistance humaine) permet de prendre la mesure des progrès accomplis. Mais le traducteur arabe est-il prêt à relever le défi posé par la traduction automatique?

Sami Trabulsi (ingénieur conseil TEAM MA ROC) a fait remarquer que si de nombreux projets sont en cours (à Damas : synthèse vocale; en Egypte projets d'analyse complète de la langue arabe; au Koweit: réaccentuation des mots arabes, etc.), bien peu ont vu le jour, faute d'investissements.

Il n'y a pas de secret, là où il y a eu investissement, il y a eu essor.

La troisième journée, consacrée à la terminologie, a mis en évidence l'absence de normalisation dans ce domaine.

Les efforts déployés par 1'IERA (Institut des études et recherches pour l'arabisation, Rabat, Maroc) sont certes importants mais leurs travaux restent peu diffusés. Il conviendrait donc, comme l'ont recommandé les participants à l'issue de cette table ronde, «d'intensifier l'effort d'unification de la terminologie à travers le monde arabe».

Harmonisation, concertation, défense du traducteur ont été les maîtres-mots de cette rencontre.

SAMIA BARRADA

École supérieure Roi Fahd de traduction, Tanger, Maroc 